\title{
Why do aged fluorescent tubes flicker?
}

\author{
Nicolas Plihon, Jérémy Ferrand, Tristan Guyomar, Flavien \\ Museur, Nicolas Taberlet \\ Univ Lyon, Ens de Lyon, Univ Claude Bernard, CNRS, Laboratoire de Physique, \\ F-69342 Lyon, France \\ E-mail: nicolas.plihon@ens-lyon.fr
}

\begin{abstract}
Common experience shows that aged and defective fluorescent tubes or bulbs may flicker and emit a clicking sound while struggling to light up. In this article, the physical mechanisms controlling the initial illumination of a functioning fluorescent tube are investigated using a simple and affordable experimental setup. Thermionic emission from the electrodes of the tube controls the startup of fluorescent tubes. The origin of the faulty startup of aged fluorescent tubes is discussed and flickering regimes using functional tubes are artificially produced when decreasing, in a controlled manner, electron emission by the thermionic effect. The physical parameters controlling the occurrence of flickering light are discussed, and their temporal statistics are reported.
\end{abstract}

\section{Introduction}

\subsection{Physical phenomenon and article outline}

Fascinating phenomena may emerge from the interplay between electromagnetism, fluid dynamics and quantum physics in plasma physics. Several experimental devices have recently been designed as educational tools at the undergraduate level $[1,2,3,4,5,6,7]$. Widely spread, fluorescent tubes or other plasma-based lighting devices remain an affordable an simple way to introduce several fundamental concepts of plasma physics [8, $9,10,11,12]$. In this article, we analyse the startup of fluorescent tubes with an emphasis on the physical understanding of the flickering operation encountered during the startup of aged or faulty fluorescent tubes.

The basic description of how fluorescent tubes operate can be found in Ref. [13, 14]. Fluorescent tubes are long glass tubes filled with noble gas (usually Argon) at low pressure (around $100 \mathrm{~Pa}$ ). When a plasma is sustained between the electrodes at each end of the tube, UV radiation from the plasma is absorbed by fluorescent coating within the glass tube, which re-emits white light.

Igniting and sustaining the plasma in the tube, while the electrodes are fed by electric power, requires external components - in particular a ballast used to limit the plasma current and an ignition-helping system known as the starter. This article focuses on the simple configuration involving a magnetic ballast and a starter, as shown in 


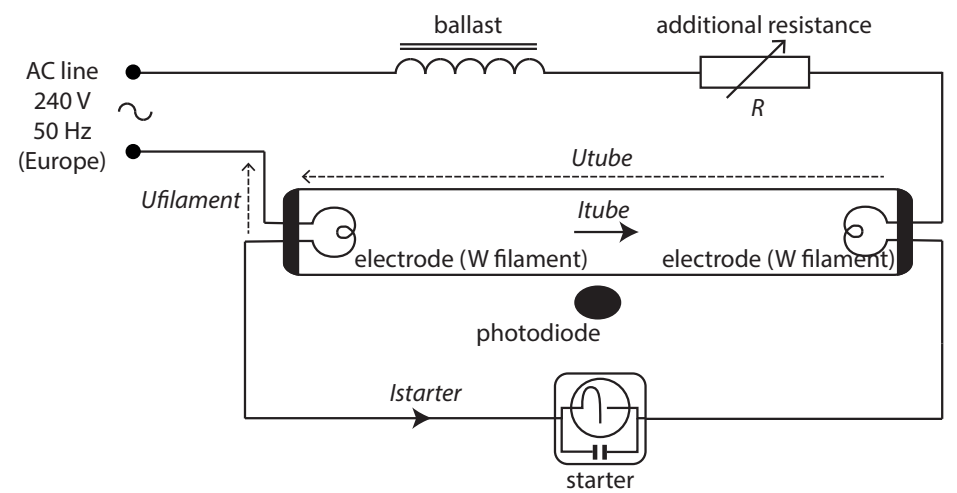

Figure 1. Schematics of the experimental setup.

Fig. 1. The electrodes (enclosed in the tube) are small foiled tungsten filaments coated with a thin layer of highly emissive oxides in order to increase electron emission from the thermionic effect. The starter consists of a separate element in which a bimetallic strip is enclosed in a low pressure noble gas capsule. When a voltage of the order of a few hundred of volts is applied across the starter, the gas it contains is ionised. The bimetallic strip heats up due to ionic bombardment on each side of the strip which act as electrodes of the starter plasma, and then deforms and closes the circuit. As a consequence a large current (around 1 A) flows through the electrodes of the tube, which are heated-up by the Joule effect. As no plasma is sustained in the (now closed) starter, the bimetallic strip cools down and eventually opens up (Joule heating in the starter is negligible as compared to the heating due to plasma bombardment). This sudden current interruption induces a large over-voltage across the large inductance of the magnetic ballast, which allows the system to reach the breakdown voltage of the noble gas enclosed in the fluorescent tube and to sustain a plasma within the tube. In this article, we provide hints that the startup of the functional tubes is controlled by the intensity of thermionic emission from the filaments. The steady-state operation of fluorescent tubes fed by the mains (240 V., $50 \mathrm{~Hz}$ in Europe, Africa or Asia or $120 \mathrm{~V}$, $60 \mathrm{~Hz}$ in the USA) involves shimmering at twice the AC line frequency [15] (100 Hz in the work reported in this article). Fluctuations of light emission of light source at the mains frequency (or its harmonics) has been studied in several contexts [16, 17]. Fluorescent tubes are based on a plasma glow discharge which is known (back to the early years of plasma physics) to be strongly intermittent [18, 19], inducing intermittent light emission. On the other hand, fluctuations of light emission of fluorescent tubes may also originate from moving non-linear potential structures in the plasma [20]. However, to the best of our knowledge, the defective startup of aged fluorescent tubes has not been reported elsewhere.

This defective startup, resulting in flickering light emission and pulses of sound emission is a widely observed phenomenon when the tube struggles to light-up. The flickering light emission appears to be random in time, with bursts of emitted light typically separated by a duration ranging from a few tenths of a second to a few seconds. 
As no steady state regime is reached, the starter continuously switches between the open and closed states, leading to the typical sound clicks (synchronised with the flickering of the tube). This peculiar regime is studied in details in this article, introducing an experimental method which reduces the efficiency of the thermionic emission. This is achieved by lowering the temperature of the filaments of functional tubes when adding an additional resistance in the electrical circuit. The article is organised as follows: plasma breakdown, plasma I-V characteristics of the glow discharge and a detailed analysis of the normal startup are described in Section 2. Section 3 then introduces an experimental setup which simulates the defective startup of fluorescent tubes resulting in random flickering light emission. The physical origin of this regime is then analysed and the statistics of the flickers are detailed. Concluding remarks are then provided in Section 4.

\subsection{What students can learn from this problem}

The work presented in this article was done in preparation for the International Physicists Tournament http://iptnet.info, a world-wide competition for undergraduate students. Unlike the typical physics exam, the problems must not only be presented, but also challenged and reviewed by the other participants allowing students to respectively assume the roles of researchers, referees and editors. In addition to the challenge that the tournament represents, it provides students with an exciting and eye-opening experience in which they learn how to design experiments with the aim of solving physics problems, and to constructively criticise scientific solutions. This article reports some of the results obtained from a series of six 4-h long labs, where students solved the question of the origin of the flickers as a problem-based learning.

The investigation of the dynamics of fluorescent tubes is a simple hands-on experiment for the introduction of plasma physics at the advanced undergraduate level. A basic understanding of electrokinetic and electromagnetism concepts and experimental techniques are required to carry this study out. Basic concepts of plasma physics, such as gas discharge ignition and sustainment, can be experimentally introduced. Other advanced such as the thermionic effect are also at grasp using this simple experiment, including the investigation of the effect of temperature on the resistance and electron emissivity of a filament. This article also provides an introduction to the statistical analysis of long time series and rare-events.

\section{Normal operation of the fluorescent tube}

\subsection{Characterisation of the fluorescent tube plasma}

Experiments have been carried out using commercial T8 types fluorescent tubes (of diameter $26 \mathrm{~mm}$ and length $590 \mathrm{~mm}$ ) Philips TL-D 18W powered from the mains $\left(U_{\text {mains }, r m s}=240 \mathrm{~V}\right.$ and $\left.f_{\text {mains }}=50 \mathrm{~Hz}\right)$ through a magnetic ballast Mazda 36N2001, as shown in Fig. 1. A Sylvania FS11 starter primes the tubes at startup. Current 
and voltages have been measured using current sensors and differential voltage sensors respectively, and the intensity of light emitted by the tube has been measured using a Thorlabs PDA10A-EC photodiode. In the remaining of Sec. 2, we investigate the normal startup operation described in the introduction. Note that the additional resistance displayed in Fig. 1 is introduced to simulate flickers (see Sec. 3) and was set to $R=0 \Omega$ for the results presented in Sec. 2.

2.1.1. I-V characteristic of the fluorescent tube plasma. Typical time series of the voltage $U_{\text {tube }}$ and current $I_{\text {tube }}$ across the tube during its steady-state normal operation are displayed in Fig. 2(a) over $20 \mathrm{~ms}$. The $I--V$ characteristics shown in Fig. 2(b) was extracted from these time series and is typical of plasma glow discharges [21], with a local negative resistance $\partial U_{\text {tube }} / \partial I_{\text {tube }}<0$. This typical $I--V$ characteristic with a large voltage required for plasma breakdown and a negative local resistance implies that sustaining the plasma in the tube is an easy task once it has been created. The negative resistance $\partial U_{\text {tube }} / \partial I_{\text {tube }}<0$ also requires current limitation, achieved by the magnetic ballast, whose resistance $R_{b}$ and inductance $L_{b}$ have been measured to be $30 \Omega$ and $1 \mathrm{H}$ respectively. This results in a ballast inductance $Z_{b}$ :

$$
Z_{b}=\sqrt{R_{b}^{2}+\left(2 \pi f_{\text {mains }} L_{b}\right)^{2}} \sim 315 \Omega
$$

larger than the tube impedance (of the order of $50 \Omega$ ), which limits the rms intensity of the current to typically $240 / Z_{b} \sim 0.8 \mathrm{~A}$.
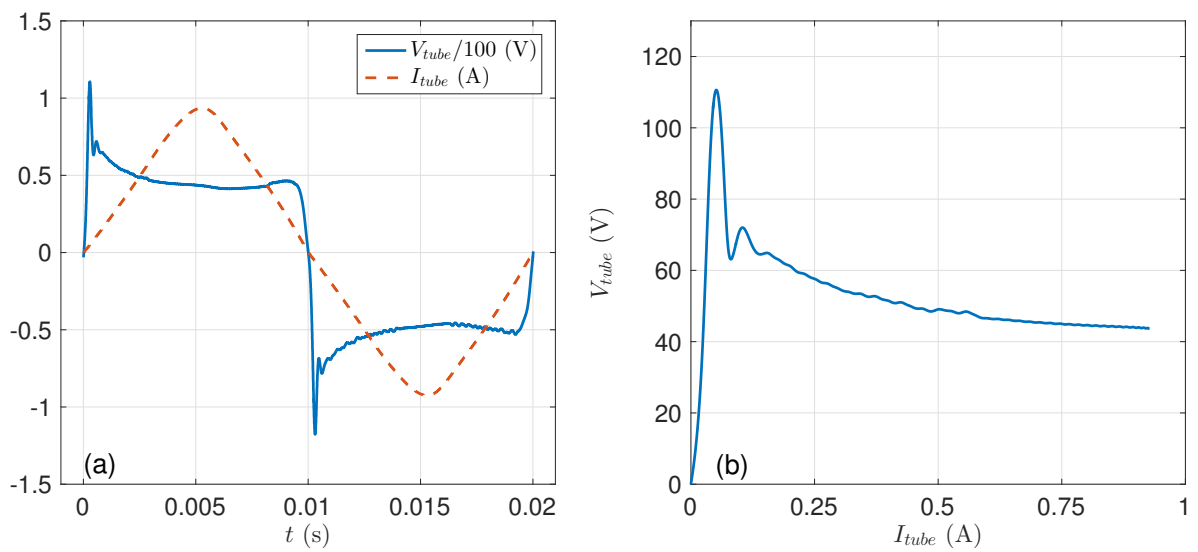

Figure 2. (a) Temporal evolution of $I_{\text {tube }}$ and $V_{\text {tube }}$ during normal operation for one $50 \mathrm{~Hz}$ cycle. (b) $I-V$ characteristics.

2.1.2. Influence of filament temperature on plasma breakdown. The ignition, or breakdown, of a plasma within a tube of length $d$ at pressure $p$ submitted to an electric field $E=U_{\text {tube }} / d$ is a well-known physical phenomenon $[5,6,21,22]$. It involves (i) exponential growth of electrons accelerated by the electric field from ionising collisions with the neutral gas, and (ii) electron emission at the cathode. At low 
cathode temperature, the latter is dominated by secondary electron emission from ion bombardment, usually characterised by a coefficient $\gamma$, which specifies the number of secondary electrons per incident particle. The voltage breakdown between two electrodes spaced apart by distance $d$ is then expressed from Paschen's law [22]:

$$
U_{b}=\frac{B p d}{\ln (A p d)-\ln [\ln (1+1 / \gamma)]},
$$

where $A$ is the saturation ionization of the gas under consideration and $B$ is related to the ionisation energy of the gas. For noble gases, both of these coefficients increase with the atomic mass [23].

When electrodes are heated up, a second process, thermionic emission, adds up to secondary electron emission. The ability of a material to emit electrons from the thermionic effect increases with its temperature $T$ following Richardson's law as $T^{2} \mathrm{e}^{-W / k_{B} T}$ with $k_{B}$ the Boltzmann constant and $W$ the work function $[24,25]$. In our experimental setup, the increase of the filament temperature $T_{\text {filament }}$ is expected to increase the value of the effective $\gamma$ coefficient and to reduce the breakdown voltage. This effect is directly measured using the dedicated setup displayed in Fig. 3(a). Both electrodes are Joule-heated by the same DC current, driven from two independent DC power supply. The measurement of the voltage $U_{\text {filament }}$ across the filament allows for the computation of the resistance of the filament $R_{\text {filament }}$ and its temperature is inferred assuming a local power balance between Joule heating and losses by radiation (an assumption valid at high enough filament temperature and low enough pressure in the tube) $[26,27]$. Assuming that the core filament is pure tungsten, the temperature is computed from the following equation $[28,29]$ :

$$
T_{\text {filament }}=T_{0}\left(\frac{R_{\text {filament }}}{R_{0}}\right)^{1 / 1.2}
$$

where $R_{0}$ is the resistance at room temperature $T_{0}$. Eq. 2 is valid above typically 150 $\mathrm{K}$ (see for instance the log-log plot in page 1092 of Ref. [30]). A high-voltage DC power supply (up to $3 \mathrm{kV}$ ) is used to bias the fluorescent tube between the hot electrodes. The DC voltage at which plasma breakdown occurs is reported as a function of $T_{\text {filament }}$ in Fig. 3(b). The filament temperature is thus shown to have a strong impact on the breakdown voltage. An abrupt decrease of breakdown voltage is evidenced above $1400 \mathrm{~K}$. This filament temperature is associated to a visual modification of the light emitted by the tube, even in the absence of a external bias between the filaments. Below $1400 \mathrm{~K}$, the light emitted by the filaments is due to thermal radiation and filaments appear reddish (see Fig. 3, bottom left photograph). Above $1400 \mathrm{~K}$, the temperature is high enough to trigger electron emission and the voltage across the filament locally sustains a plasma; UV radiated by the plasma excite the phosphor coating, which in turn emits white light (see Fig. 3, bottom right photograph). 

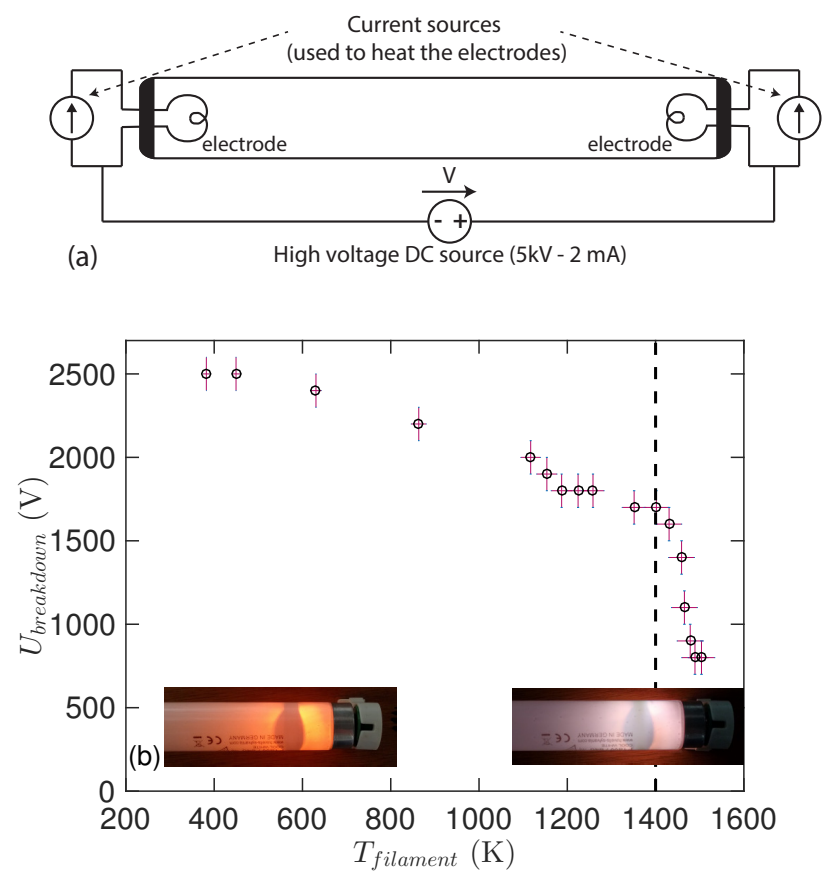

Figure 3. Influence of filament temperature on breakdown voltage: (a) experimental setup, (b) voltage breakdown as a function of $T_{\text {filament }}$. The vertical dashed line indicates the visual modification of the tube. See text for details.

\subsection{Time analysis of normal startup}

Let us now investigate the temporal dynamics of normal startup as the electric power of the tube is fed by the mains as displayed in Fig. 4. At time $t=0$, the mains are switched on: the line voltage (rms voltage of $240 \mathrm{~V}$ ) is applied to the tube and the starter, but no current flows. At $t \sim 0.05 \mathrm{~s}$ (identified by vertical lines in Fig. 4), the gas enclosed in the starter capsule becomes ionised: a weak current of the order of 50 $\mathrm{mA}$ crosses the starter during the positive voltage half-wave and the bimetallic strip enclosed in the starter heats-up. At $t \sim 0.31 \mathrm{~s}$ (identified by vertical lines in Fig. 4 ) the deformation of the bimetallic strip is large enough to close the electrical circuit (referred to as closed starter in the remainder of this paper) and a current of typically 1 A flows in the starter and the filament electrodes. This large current leads to Joule heating of the filaments, while the starter cools down (as no plasma is present in the starter). Finally at $t \sim 0.88 \mathrm{~s}$ (identified by vertical lines in Fig. 4 ) the starter opens, leading to a large over-voltage across the tube, exceeding the breakdown voltage and igniting the plasma in the tube. Note that light emission is modulated at $100 \mathrm{~Hz}$, with maxima for each half-wave of the mains voltage [15].

The startup process thus strongly relies on the pre-heating of the electrodes when the starter is closed. The temporal evolution of the current flowing in the starter, the filament resistance and the filament temperature during the pre-heating phase is displayed in Fig. 5. The filament resistance was measured as the ratio of the envelope 


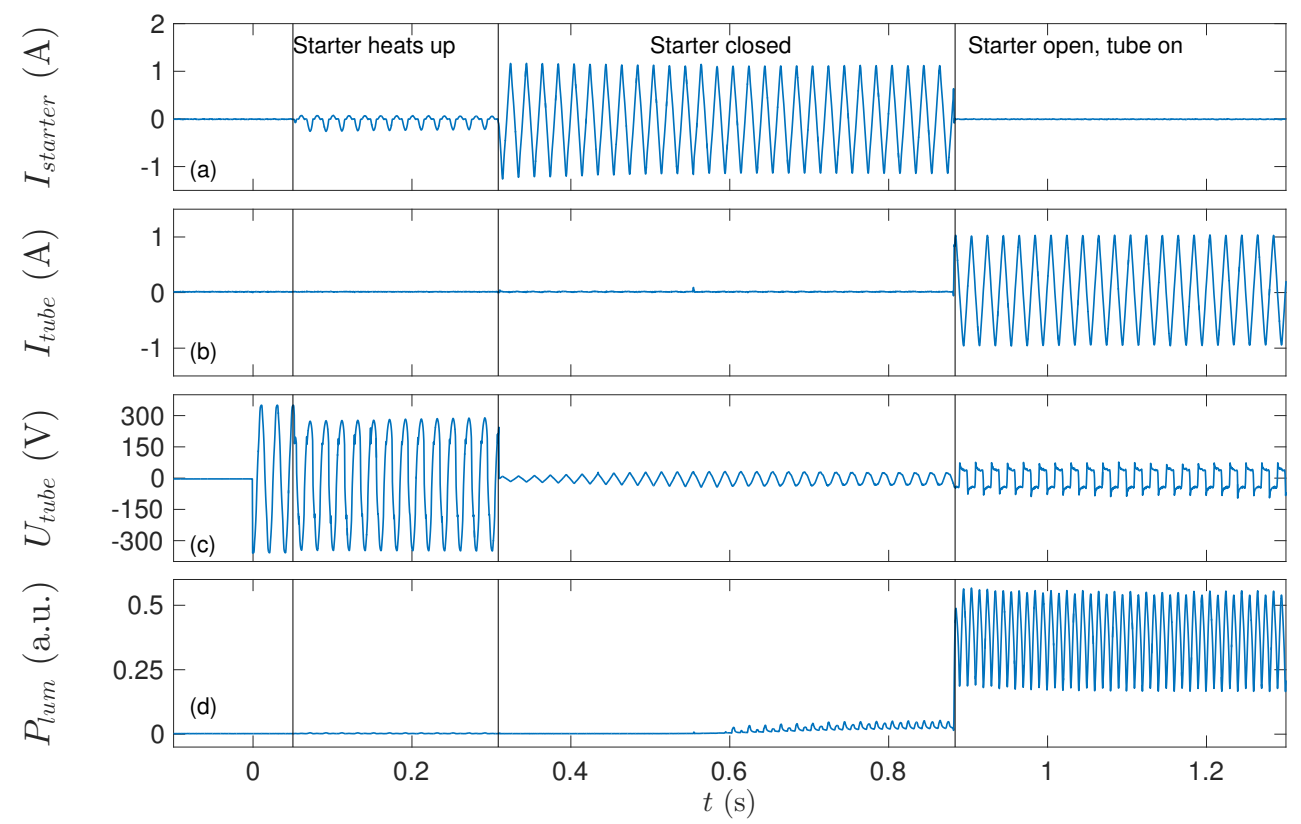

Figure 4. Time series of (a) starter current (or filament current) $I_{\text {starter }}$, (b) current in the tube $I_{\text {tube }},(\mathrm{c})$ voltage across the tube $V_{\text {tube }}$ and (d) light intensity $P_{\text {lum }}$ showing a typical tube startup time sequence of a tube initially at ambient temperature. See text for details.

of the filament voltage over the envelope of the starter current (this is valid as long as no plasma is present in tube, i.e. when $I_{\text {tube }}=0$ ), and reaches a few tens of Ohms (error bars are displayed as the grey area). The resistance of both electrodes (typically less than $50 \Omega)$ remains at least five times lower than the ballast impedance $\left(Z_{b} \sim 315 \Omega\right)$; as a consequence the amplitude of the current flowing in the filament remains roughly constant during pre-heating, while the voltage across the filament increases (not shown). The time evolution of the filament temperature $T_{\text {filament }}$, computed from Eq. 2 , is displayed in Fig. 5(c). After a few tenths of seconds of preheating, $T_{\text {filament }}$ reaches values around $1500 \mathrm{~K}$, i.e. at the onset of emission by the thermionic effect, which decreases the breakdown voltage to ignite the plasma, as described in subsection 2.1.2.

\section{Flickering regimes}

Flickering regimes observed at the startup of aged fluorescent tubes involve faulty startups, i.e. plasma breakdown is reached transiently but the plasma within the tube is not steadily sustained. From the previous observations, this is likely to be related to inefficient pre-heating of the filaments. In aged fluorescent tubes, thermionic emission might be decreased from sputtering of the filament emissive layer, oxidation of the emissive layers, or deposition of other materials on these emissive layers. Note that sputtering or evaporation of the filaments would lead to a gradual decrease of 


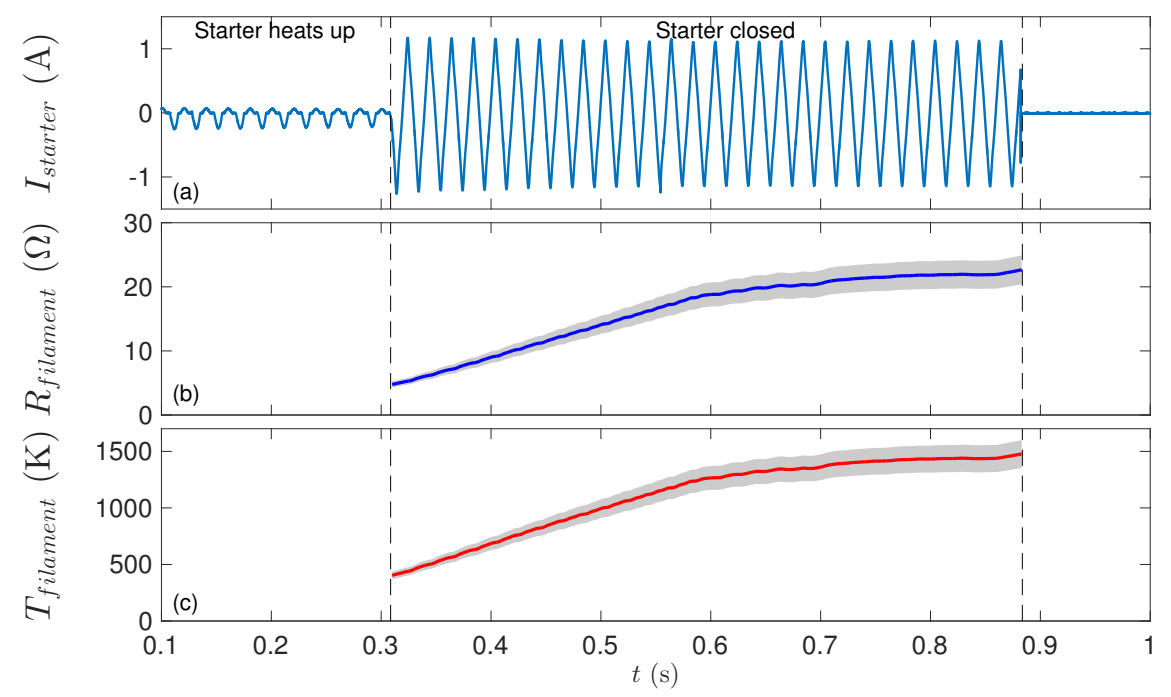

Figure 5. Time evolution of (a) starter current (or filament current), (b) $R_{\text {filament }}$ and (c) $T_{\text {filament }}$ during pre-heating phase, when the starter is closed. Error bars displayed as grey areas. See text for details.

the filaments' diameter and to a gradual increase of $R_{\text {filament }}\left(T_{0}\right)$. However, this effect is likely to be counter-balanced by the sputtering of the strongly emissive layers: the thermionic effect from the bulk filament material is likely to be lower than that from the emissive layer. On the other hand, modification of the gas composition could also lead to a change of breakdown voltage. Ageing of the starter could also lead to the decrease the duration of closed time, which would then lead to less efficient pre-heating. However, we postulate that the leading process controlling the tube ignition is thermionic emission from the electrodes. In this framework, faulty startups using functional fluorescent tubes can be obtained when decreasing the temperature of the filament (since thermionic emission depends exponentially on the material temperature according to Richardson's law). In order to experimentally simulate the inefficient pre-heating of the filaments, an additional high-power resistance $R$ was inserted in series with the ballast, as shown in Fig. 1. This additional resistance reduces the amplitude of the current flowing in the filaments, which lowers the efficiency of the preheating. An order unity decrease of the current flowing in the filaments has a strong effect on the Joule heating of the filaments, which in turn has a strong influence on the thermionic effect. As a consequence, the value of the additional resistance $R$ was chosen to be a few times that of the ballast's impedance $Z_{b} \sim 315 \Omega$. Flickering light emission from the tube was observed for $R$ values in the range 500-1000 $\Omega$. For $R$ values greater than $1000 \Omega$, no light emission was observed. Note that similar results have also been observed when decreasing the voltage amplitude feeding the circuit with $R=0$ (for instance using an autotransformer between the mains and the circuit), which also reduces the current flowing in the filaments during the pre-heating phase. 


\subsection{Description of flickering regimes}

Several flickering regimes have been observed depending on the value of the additional resistance $R$ and typical time series of light emission are reported in Fig. 6 (for the sake of clarity, time-series are vertically shifted). The regime shown in Fig. 6(a) consists in a random distribution of short pulses, and was observed for $R$ values in the range 910 to $550 \Omega$. In this regime, the plasma is sustained only during a fraction of a single halfperiod of the mains $50 \mathrm{~Hz}$ cycle: a single peak of light emission is observed before fading out. As the value of $R$ is decreased, light emission is no longer limited to short pulses, as shown in Fig. 6(b-e). The plasma may be sustained in the tube during several mains cycles, leading to a flickering regime consisting in a train of pulses of light emission. Note that the light is $50 \mathrm{~Hz}$ modulated during one flicker, which is highly uncomfortable to the human eye - it is shown later that the plasma is only sustained during a given polarity of the mains. Successive flickers may be excited as reported in Fig. 6(b) and (c) or may alternate with short pulses as reported in Fig. 6(e). Finally, for $R$ values lower than 500 $\Omega$, the flickering regime slowly drifts and finally leads to normal operation of the tube as shown in Fig. 6(f). Note that in this last regime, and for times larger than $0.2 \mathrm{~s}$, the light is $100 \mathrm{~Hz}$ modulated as during normal operation (however, the introduction of the additional resistance $R \sim 1.5 Z_{b}$ reduces the light intensity by a factor around 2.5).

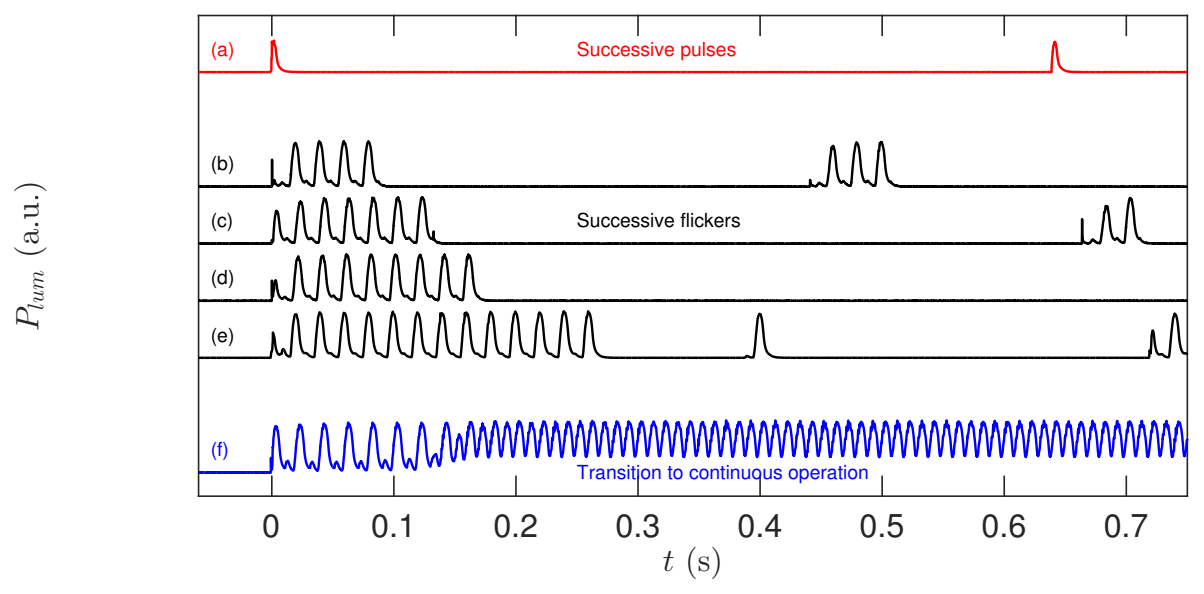

Figure 6. Typical time-traces of flashes observed as $R$ decreases from 910 to $457 \Omega$ (top to bottom). See text for details.

The detailed analysis of the regime reported in Fig. 6(f) is instructive. Simultaneous time series of light intensity $P_{\text {lum }}$, voltage across the tube $V_{\text {tube }}$, and plasma current $I_{\text {tube }}$ are reported in Fig. 7. For $0<t<0.12 \mathrm{~s}$ significant light emission is only observed when the voltage across the tube is positive: the plasma is only sustained during a given polarity of the $50 \mathrm{~Hz}$ cycle, resulting in $50 \mathrm{~Hz}$ modulation of light emission. This is likely to originate in the efficient pre-heating of only one of the two filaments, say electrode 1. During the positive voltage half-wave, plasma breakdown is obtained when 
electrode 1 acts as the cathode, while breakdown is not reached when electrode 1 acts as the anode, i.e. during the negative voltage half-wave (not that the scale of Fig. 7(b) is not symmetric). It is important to note that, since thermionic emission exponentially increases with temperature according to Richardson's law, a very weak temperature difference between both electrodes leads to a large difference in current emitted by the thermionic effect. Weak geometric or assembly asymmetries between both electrodes are thus expected to result in strong difference in thermionic emission at onset. However, as electrode 2 is further heated by ion bombardment and current injection into the plasma, it acts as a more and more efficient cathode for $0.12<t<0.18 \mathrm{~s}$. Finally a symmetric behaviour is recovered for times larger than $0.2 \mathrm{~s}$, resulting in a $100 \mathrm{~Hz}$ modulation of light emission as reported in Section 2.2. The asymmetric regime may thus only be obtained in a narrow range of parameters.

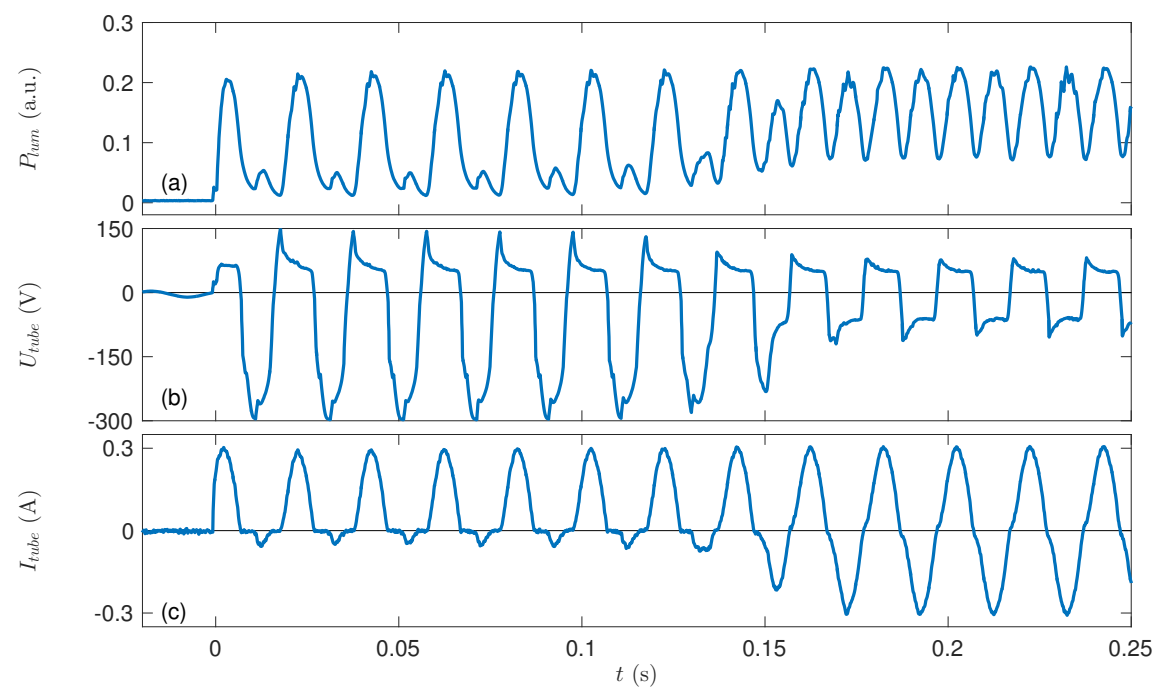

Figure 7. Time evolution of (a) light intensity $P_{\text {lum }}$, (b) voltage across the tube $V_{\text {tube }}$, (c) the plasma current $I_{\text {tube }}$ for the regime reported in Fig. $6(\mathrm{f}), R=457 \Omega$. See text for details.

\subsection{Time statistics of flickers}

3.2.1. How flickers onset are related to the opening of the starter. The common experience with aged and flickering fluorescent tubes links flickering light emission and sound emission from the starter. As long as no plasma is sustained in the fluorescent tube, the voltage across the starter is high enough to reach breakdown of the starter gas: as a consequence, the starter continuously alternates closed and open phases. Plasma breakdown in the tube necessarily occurs at the opening of the starter, as shown in the previous Section. However, as shown in Fig. 8(a), light emission pulses do not systematically coincide with the opening of the starter (shown as red lines in the top panel). In our experimental setup, the inefficient pre-heating of the filaments requires the opening of the starter within a short period of the $50 \mathrm{~Hz}$ mains to ensure plasma 
breakdown in the fluorescent tube. Indeed, plasma breakdown requires a high enough over-voltage across the ballast (which depends on the value of the current at the opening time), and during the positive polarity of the $50 \mathrm{~Hz}$ mains voltage (as described in the previous subsection). For these reasons, plasma breakdown is not observed for all opening events of the starter. The filament temperature $T_{\text {filament }}$ is reported in Fig. 8(b), and shows that a stationary regime for the filament temperature is reached, allowing for the statistical analysis of flickering regimes. The regime reported in Fig. 8 consists of single pulses, but it is important to note that, for longer flickers, the opening of the starter is not systematically associated to light emission. The time-distribution of light flickers is investigated in details in the next two subsections; for the sake of simplicity, the temporal dynamics of the opening/closing sequences of the starter is not investigated explicitly.
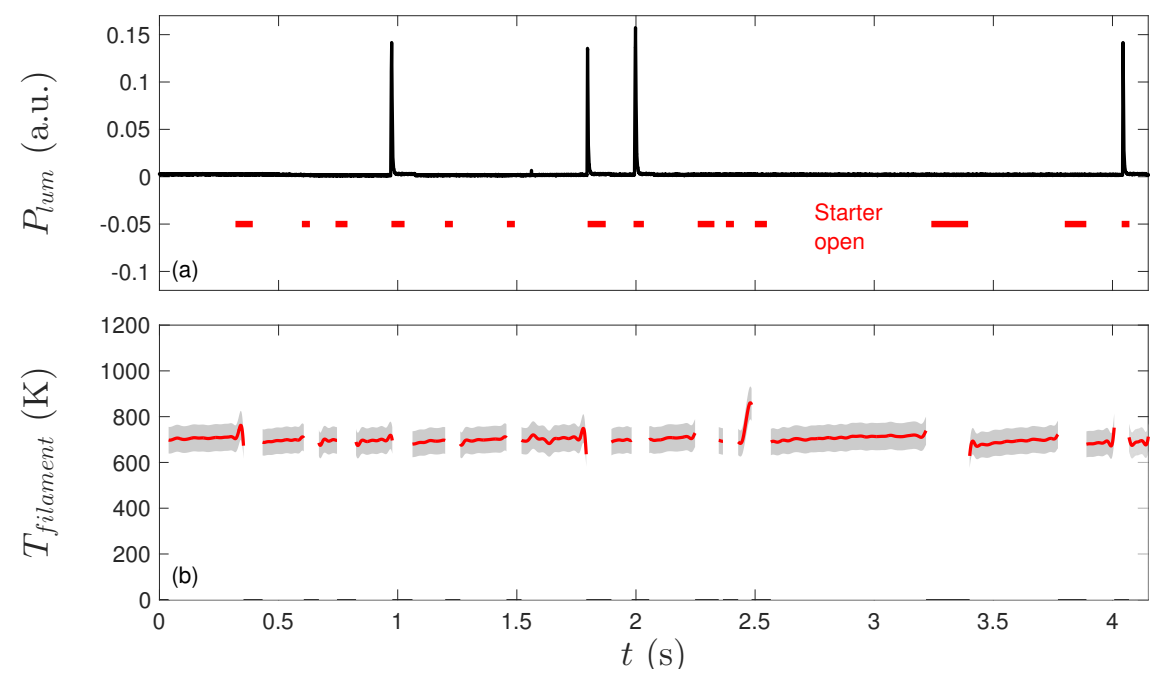

Figure 8. Time evolution of (a) light intensity and (b) filament temperature $T_{\text {filament }}$. Open starter phases identified by red lines on panel (a).

3.2.2. Statistics of single pulses. Let us first investigate the statistics of successive single pulses (i.e. the regime reported in Fig. 6(a)) for $R$ values in the range [550; 910] $\Omega$. Pulses typically last less than $5 \mathrm{~ms}$ and do not strongly change from one to the other, we thus focus our attention on the distribution of the waiting-times between two successive pulses. One hour-long acquisitions were acquired for several values of the additional resistance $R$. The photodiode signal is used to trig a fast oscilloscope (with a $5 \mathrm{~ms}$ time base) and the digital TTL trigger-out signal of the oscilloscope is acquired at a rate of $250 \mathrm{~Hz}$. The probability distribution functions (pdf) of the waiting-times are displayed in Fig. 9(a) for three values of $R$ and are consistent with exponential distributions. The pulses thus appear to be randomly distributed, without any correlation between occurrences. The characteristic mean waiting-time $\langle\tau\rangle$ between two pulses times weakly evolves with $R$ : $\langle\tau\rangle$ decreases from $0.62 \mathrm{~s}$ to $0.57 \mathrm{~s}$ and finally $0.51 \mathrm{~s}$ as $R$ decreases from 
910 to 700 and finally $550 \Omega$ respectively. It is important to note that this decreasing trend is observed since the tails of the pdf are fairly well resolved. As the value of $R$ decreases, the current in the pre-heating phase increases, and the plasma is more likely to ignite: the increase of the filaments's temperature make them more emissive and the increase of the current increases the over-voltage $L_{b} d I / d t$ across the magnetic ballast. As a consequence, the mean waiting-time slowly decreases with decreasing values of $R$.

3.2.3. Statistics of longer flickers. Let us now investigate the statistics of the longer flickers obtained when $R<550 \Omega$, i.e. the regimes reported in Fig. 6(b-e) where both short pulses and longer flickers are present. For a typical configuration with $R=497 \Omega$ nearly 700 light emitting events have been measured over $1800 \mathrm{~s}$, out of which $61 \%$ are long flickers (lasting at least two mains cycles). Note that these regimes with long flickers are extremely unstable: a slow time-evolution of the filament temperature could lead either to recover a symmetric behaviour leading to continuous light emission or to recover regimes consisting in a succession of short pulses. Our study focusses on two distinct probability distribution functions: namely the pdf of the duration of on-phases (i.e. the duration of the flicker light emission), and the pdf of the duration of waiting times (or equivalently off-phases). The distribution of waiting times, shown in Fig. 9(b), is monotonically decreasing, while the distribution of on-phases, shown in Fig. 9(c), is clearly peaked with a most probable value around $0.7 \mathrm{~s}$ and a long tail towards large values. Note finally that the distributions shown here should be regarded as illustrative due to the unstable behaviour in this range of parameters (the fluorescent tube was finally continuously emitting light at the end of the time series under investigation). As a consequence, the deviation of the waiting-times distribution from an exponential distribution might be due to the non-stationarity of the regime - since the physical origin of the flicker onset is the same as for the pulses, one would expect the distributions to be similar. Addressing this issue would require a careful control of the stability of the flickering regime.

\section{Conclusion}

The physical processes controlling the startup of fluorescent tubes used with a magnetic ballast and a starter were investigated experimentally, using an inexpensive and easy to reproduce experimental setup. The ability of the electrodes at each end of the fluorescent tube to emit electrons is shown to be the leading parameter controlling the voltage required across the fluorescent to reach plasma breakdown. In particular, for a given tube assembly, electron emission from thermionic emission is controlled by the temperature of the electrodes. A detailed time sequence of the startup of fluorescent tubes is reported, including detailed experimental measurements of the electrode temperature. Finally the inability of aged fluorescent tubes to undergo quick ignition is experimentally addressed. In this regime, light emission flickers and random noise emission are experienced at startup. An experimental method to simulate 

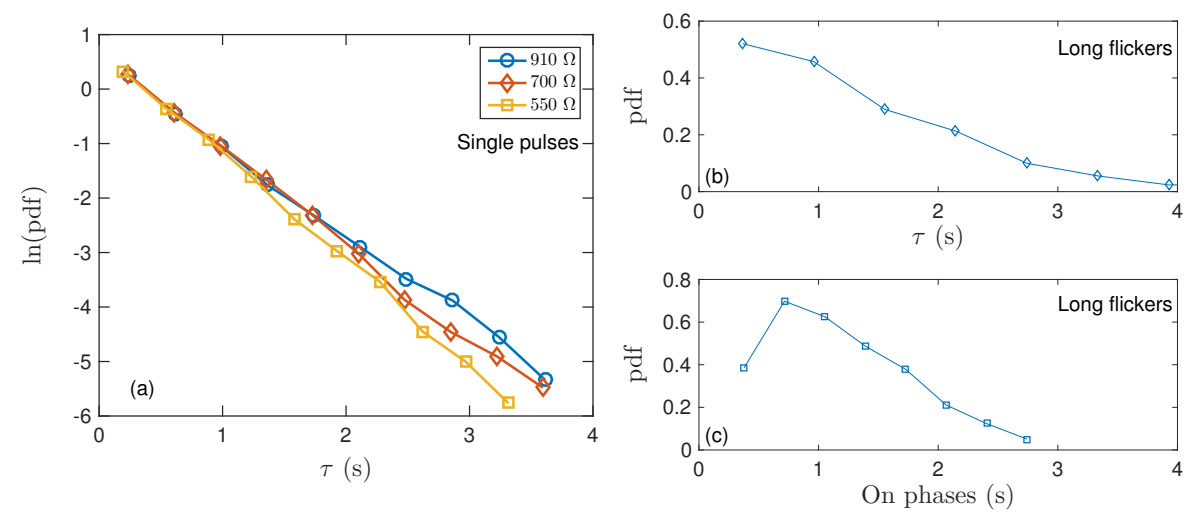

Figure 9. (a) Probability distribution function of waiting times $\tau$ for the successive pulses regime reported in Fig. 6(a) for three values of $R$. Probability distribution functions of (b) waiting times $\tau$ and (c) on-phases for the long flickers reported in Fig. 6(e).

flickering regimes using standard tubes is introduced. This relies on reducing the current heating the electrodes when the starter is closed since the flickering regime originates in inefficient pre-heating of the electrodes which only transiently sustains the plasma in the fluorescent tube. In particular, in our experimental setup, flickers are observed when only one of the electrode reaches a temperature high enough to strongly emit electrons when negatively biased. Using this method, statistically stationary flickering regimes are observed, allowing for the statistical analysis of the time distribution of flickers. The onset of flickers appear to be random in time, with no correlation, resulting in an exponential probability distribution function.

\section{Acknowledgments}

This work was supported by the ś Normale Supérieure de Lyon and Univ Claude Bernard, Lyon, France. The authors acknowledge J. Bernard, E. Camus, L. Lachaud, Q. Marsal and L. Thorens for fruitful discussions. The authors are grateful to both local and international organizing committees of the 2017 International Physicists Tournament for having put together an exciting event.

\section{References}

[1] Gekelman W. et al. 2007 Ion acoustic wave experiments in a high school plasma physics laboratory Am. J. Phys. 75 103-110

[2] Lisovskiy V and Yegorenkov V 2009 Validating the collision-dominated Child-Langmuir law for a DC discharge cathode sheath in an undergraduate laboratory Eur. J. Phys. 30 1345-1351

[3] Gekelman W. et al. 2011 Using plasma experiments to illustrate a complex index of refraction Am. J. Phys. 79 894-902

[4] Lisovskiy V A, Koval VA, Artushenko E P and Yegorenkov V D 2012 Validating the Goldstein- 
Wehner law for the stratified positive column of DC discharge in an undergraduate laboratory Eur. J. Phys. 33 1537-1545

[5] Wissel S. A., Zwicker A., Ross J. and Gershman S. 2013 The use of DC glow discharges as undergraduate educational tools Am. J. Phys. 81, 663-669

[6] Lisovskiy V and Yegorenkov V 2014 In-depth treatment of discharge ignition data during undergraduate laboratory work Eur. J. Phys. 35045021

[7] Gekelman W. et al. 2016, Drift waves and chaos in a LAPTAG plasma physics experiment Am. J. Phys. 84 118-126

[8] Auerbach I. 1961, Fluorescent Lamp. A Teaching Device Am. J. Phys. 29, 546-547

[9] Field A. and Wolfson R. 1987, Microwave measurements of a fluorescent lamp plasma Am. J. Phys. 55, 637-641

[10] Orr J. and Wolfson R. 1990 Cyclotron resonance effects in a fluorescent lamp plasma Am. J. Phys. 58, 968

[11] Nassar A. and Mednik M. 2003, Introductory physics of harmonic distortion in fluorescent lamps Am. J. Phys. 71, 577-579

[12] Eldakli M S A, Ivković S S and Obradović B M 2017, Optogalvanic effect and laser-induced current oscillations in hollow-cathode lamps Eur. J. Phys. 38025210

[13] Bloomfield L. A. 2016, How things work: the physics of everyday life, John Wiley and sons ed (New-York), 6th edition

[14] Lister G. G., Lawler J. E., Lapatovich W. P. and Godyak V. A. 2004, The physics of discharge lamps Rev. Mod. Phys. 76 541-98?

[15] Vollmer M and Möllmann K-P, 2015 Flickering lamps Eur. J. Phys. 36035027

[16] Bacalla X and Salumbides E J 2013, On the intensity profile of electric lamps and light bulbs Phys. Teacher $\mathbf{5 1}$ 491-2?

[17] Andersson N, Sandstrm M, Berglund A and Hansson K 1994, Amplitude modulation of light from various sources Lighting Res. Tech. 26 157-60

[18] Kock A. E. 1933, The Effect of Inductance on the Intermittent Glow Discharge Physics 4, 359

[19] Boyle W.S. 1955, SelfPropagating Intermittent Discharge J. Appl. Phys.26, 584

[20] Liu Y, Chen D, Buso D, Bhosle S and Zissis G 2008, Experimental investigations on moving striations in a $50 \mathrm{~Hz}$ ac fluorescent lamp J. Phys. D: Appl. Phys.41 135211

[21] Raizer Y. P. 1991, Gas discharge physics, Springer-Verlag ed. (Berlin)

[22] Yip C.-S. and Severn G. 2015, Comment on "The use of dc glow discharges as undergraduate education tools" Am. J. Phys. 83654

[23] Lieberman, M.A. and Lichtenberg A.J. 2005, Principles of plasma discharges and materials processing. Wiley, New Jersey

[24] Azooz A A 2007 An experiment on thermionic emission: back to the good old triode Eur. J. Phys. 28 635-642

[25] Kittel C 1966 Introduction to Solid State Physics, 3rd ed, Wiley (NewYork)

[26] Carlà M 2013, Stefan-Boltzmann law for the tungsten filament of a light bulb: Revisiting the experiment Am. J. Phys. 81, 512

[27] de Izarra C and Gitton J.-M. 2010 Calibration and temperature profile of a tungsten filament lamp Eur. J. Phys. 31 933-942

[28] Zanetti V. 1985, Temperature of incandescent lamps Am. J. Phys. 53, 546

[29] Prasad B. S. N. and Mascarenhas R. 1978, A laboratory experiment on the application of Stefan's law to tungsten filament electric lamps Am. J. Phys. 46, 420

[30] Desai P.D, Chu T.K., James H.M. and Cho C.Y. 1984, Electrical Resistivity of Selected Elements J. Phys. Chem. Ref. Data 13, 1069-1096 